\title{
Adopting the intentional stance towards humanoid robots
}

\author{
Authors: Jairo Pérez-Osorio \& Agnieszka Wykowska
}

Istituto Italiano di Tecnologia, Genova, Italy

\begin{abstract}
On the day by day humans need to predict and understand others' behavior in order to efficiently navigate through our social environment. When making predictions about what others are going to do next, we refer to their mental states, such as beliefs or intentions. At the dawn of a new era, in which robots will be among us at homes and offices, one needs to ask whether (or when) we predict and also explain robots' behavior with reference to mental states. In other words, do we adopt the intentional stance (Dennett, 1987) [1]) also towards artificial agents-especially those with humanoid shape and human-like behavior? What plays a role in adopting the intentional stance towards robots? Does adopting an intentional stance affect our social attunement with artificial agents? In this chapter, we first discuss the general approach that we take towards examining these questions-using objective methods of cognitive neuroscience to test social attunement as a function of adopting the intentional stance. Also, we describe our newly developed method to examine whether participants adopt the intentional stance towards an artificial agent. The chapter concludes with an outlook to the questions that still need to be addressed, like ethical consequences and societal impact of robots with which we attune socially, and towards which we adopt the intentional stance.
\end{abstract}

Keywords: intentional stance, social robotics, human-robot interaction, mental states

\section{Introduction}

The ability to comprehend beliefs, desires or intentions of others is characteristic of, and necessary for, successful human interaction. It enables sharing mental representations, building common goals and acting in unison. Thanks to cognitive mechanisms specialized in perceiving social signals, humans and other primates are relatively good in anticipating others' behavior, inferring mental states and taking others' perspective. The strategy of referring to others' mental states in order to predict their behavior has been termed "adopting the intentional stance" [1]. This strategy is very quick and is adopted spontaneously. It generates predictions that adapt to different contexts, it is relatively efficient and accurate, and it works despite a lack of knowledge regarding the physical complexity underlying behavior (for example, neuronal activity).

Facing the prospect of introduction of robots into society, the question about social dynamics during interaction with humanoid robots emerge. It is plausible to think that the same social cognitive mechanisms that allow social attunement in human interactions might be activated during human-robot interaction (HRI). Currently, robot designers have been focusing on providing the robots with human-like appearance, behavior, or communication skills. This has generated mixed results.

In this chapter, we argue that adopting the intentional stance might be a pivotal factor facilitating social attunement with artificial agents. We provide a summary of Dennett's idea of the intentional stance, we review literature related to attribution of intentions and mental states from the developmental and cultural perspectives, and then provide considerations regarding the foundations of the intentional stance and how this translates into human-robot interaction. The last part of the chapter is dedicated to the discussion regarding ethical implications of adopting the intentional stance towards humanoid robots.

\section{The three stances}

The brain has evolved to discriminate patterns and regularities in order to predict future states and events. From predicting a storm to understanding when others are bidding for attention, this "active perception" allows efficient functioning in complex environments. Humans use different strategies to understand and predict events. Dennett $[1,2]$ postulated three main strategies, dependent on the observed systems. For some systems, predictions are based primarily on the laws of physics and on the physical properties of things. Interactions between celestial bodies is an example. Such approach is very accurate because it relies on the exhaustive understanding of most of the crucial variables that intervene and interact on the system. When we make predictions depending on the physical properties of the system, we adopt the physical stance. 
The main drawback of the physical stance is that it is impractical for certain systems. For those systems, predictions that refer to their intended functionality or design are more efficient. Think of a bicycle. It is not necessary to know how torque or gyroscopic precession keep the bicycle balanced, to predict its behavior. However, we know that if we keep on pedaling, it will stay in balance. In this case, when we generate predictions based on the design characteristics of the system we adopt the design stance. These predictions rely on additional assumptions or previous conventional knowledge regarding the purpose of the design. In other words, it depends on non-intuitive information. Nevertheless, the design stance pays off. Once we know the purpose of an artefact, predictions become highly reliable and very practical because they circumvent the intricacies of the physical stance. The design stance can be applied to artefacts designed by humans or to living things designed by evolution. It can describe the behavior of animals, plants, organs or even a biome.

The behavior of a certain class of complex living systems (humans) is best explained with reference to yet another stance, the intentional stance. According to Dennett, these are true intentional systems, as for them, intentional stance works best. It is the most efficient strategy to understand and predict the behavior of true intentional systems. The intentional stance is the strategy of interpreting the behavior of an entity by treating it as a rational agent who makes behavioral choices in line with their desires and goals, and ways that s/he believes will lead to the achievement of the set goals [3]. Similar to the design stance, the intentional stance is a gamble that pays off in general. Thousand years of evolution designed human beings to be rational, to believe what they ought to believe and want what they ought to want. The fact that we are products of a long and demanding evolutionary process guarantees that using the intentional strategy on ourselves is a safe bet. At its core, adopting the intentional stance is a bet for rationality. It implies that people's behavior is ultimately regulated by their decisions and the capacity to execute them. For example, people exercise because: they want to be healthy, they desire to be in shape and they believe that exercising will lead to being healthy and in shape.

Dennett borrowed the terms "belief" and "desire" from folk psychology but he gives them a more technical meaning. For him, beliefs and desires are examples of propositional attitudes - mental states that have the property of "aboutness". He claims that the physical instantiation of these intentional states is irrelevant to the theory's predictive efficacy. Dennett claims that any system whose behavior can be best predicted by the intentional stance can be considered an intentional system. Furthermore, he suggests that intentional stance is a craft, a practice of folk psychology, very useful to predict behavior and used in an intuitive and automatic fashion.

\section{Attribution of mental states to events and objects}

Humans are capable of attributing human-like characteristics to pretty much anything. Oral tradition and records from earlier civilizations and cultures reveal this tendency to anthropomorphize anything that shows apparent independent agency: animals, natural events like storms or volcanos, the sun and the stars. Even today, this predisposition seems to have remained. Human-like motivations, intentions and mental states might be attributed to electronic or mechanical devices, computers, or in general anything that gives the impression of an autonomous agency. The level of attribution can involve assumption of mind, such as conscious experience, metacognition, and intentions [4], emotional states, behavioral characteristics, or human-like forms to non-human agents and to inanimate objects ([5]; also reviewed in [6]). Psychologically, anthropomorphism is considered a default and automatic psychological process (see [7]) that is often employed towards systems whose behavior we do not understand, as it is the most available and familiar explanatory mode ([6], [8], [9]). Interestingly, studies showed that anthropomorphism relies on the same cognitive mechanisms that generate attribution of intentions to human behavior ([10]; [11]).

\section{Intentional stance and development}

Humans are very skilled at interpreting actions in terms of mental states. This skill seems to be acquired from a very young age. Therefore, developmental psychology is interested in explaining the emergence of this ability. However, one argument against the idea that already infants adopt intentional stance is that typically, children pass the explicit verbal false belief test only when they are four years old or older [12-14]. Therefore, it seems unlikely that children ascribe to others beliefs, desires and rational thought processes in early childhood. Nonetheless, multiple developmental studies have examined the cognitive mechanisms closely related to adopting the intentional stance before the robust appearance of the theory of mind (ToM) [12]. 
For example, five-month-old infants seem to have shown goal-attribution to objects that exhibit cues of animacy [15]. By six months, infants' gaze following reveals sensitivity to attentional states [16] -which is deeply linked to the appearance of affect attunement (sensitivity to others' emotions) [17]. At the same age, infants perceive goal-related movements on the part of geometric shapes [18]. Recognition of gaze direction together with the recognition of animacy of movements, enable developing associations between agents' behaviors and respective contingent effects in the environment, which leads to an early causal understanding of the world. Therefore, actions in familiar contexts begin to generate expectations. From 6 months of age, infants show the development of at least a basic mentalizing ability. They can differentiate pretended versus intended actions (e.g., [19]), also pretended versus intended but impossible [20], and can identify individuals' preferences (e.g., [21]). By 9 months of age, infants understand that others have goals, that they are committed to achieving them and that different outcomes are linked to particular emotional states (i.e., success $=$ happiness, or at least smiles) [22]. Around ten months, they parse streams of behavior into units that correspond to what adults would see as separate actions [23].

At one year of age, crucial social behavior emerges. Communicative gestures (i.e., pointing) and gaze following, both necessary for shared attention, become part of the children behavioral repertoire. Furthermore, detecting these social signals influences children's course of action ([24]; [25]). This suggests that the infant is coding the adult's mental state of attention to, or emotion about, a state of affairs. At this age, infants reliably follow the gaze of a faceless animal-like object if the object reacts contingently on their behavior [26]. Also, one-year-olds can differentiate whether a behavior is goal-directed based on the context in which behavior unfolds. Fourteen- to eighteen-month-old children will imitate a goal that has not been achieved by another person if the action is marked linguistically as purposeful, but not if it is marked as accidental [27]. By 14 months of age, infants infer others' intentions and goals [28] and can set aside their own desire, recognize the others' different desires and act accordingly [21]. At the same age, infants start understanding that behavior unfolds following goal-oriented action plans and that attending to the goalrelated elements of the action sequence is crucial for goal accomplishment. Such understanding builds the foundation for imitative learning, a powerful form of cultural learning. Intention understanding not only allows predicting what others will do but also learning from them how to do things conventionally in their culture [29].

Two-year-old children appear to be capable of drawing pretended consequences from pretended assumptions. Harris [30] found that two-and-a-half-year-old children can distinguish between a pretend and a real outcome from a pretend or real assumption (e.g., that chocolate would be wet/dry after having pretend/real tea poured on it). By 3 years of age, children start to understand the role of beliefs in intentional action and more accurately grasp the connections among desires, perceptions, and emotions [22]. Some researchers suggest that children around 4 years of age can predict behavior based on a false belief (in a novel situation) ([12]; [31]). Shortly after learning to speak, kids use successfully simple mental states terms like want, pretend, know, and think [32]. However, 4- and 5-year-old children are unable to appreciate that, in order to pretend, an actor must have the intention to pretend [33], and they struggle to distinguish between intentions and desires that relate to the same goal [34]. Children's well-documented success in falsebelief tasks at around 4 years of age (e.g., [31]) is typically taken as evidence of their ability to represent mental states as such - an ability that applies to intentions just as it does for beliefs [35]. However, children's conceptual understanding of intentions -of the motivational, causal, and epistemic componentsbegins before this age and continues afterward [36].

In a review of cues that might trigger attribution of intentionality during infancy, Johnson [37] highlights five main characteristics: (1) features like face and eyes; (2) an asymmetry along one axis, like having a head smaller than the body; (3) non-rigid transformations such as expansion and contraction, contrary to linear changes; (4) self-propelled movement; and (5) the capacity for reciprocal and contingent behavior. However, it remains unclear how the combination of these features and their interactions with the environment triggers attribution of intentionality, as not all asymmetrical or self-propelled agents evoke such attributions [38].

In summary, studies reveal that infants are able to use the context, the observed behavior and social signals to understand that others' actions are a result of mental states such as intentions. This skill appears by the end of the first year and gets robust around 18 months. It is clear that probably infants do not adopt the intentional stance in its fullest sense, as defined by Dennett $[1,39]$. Nonetheless, developmental findings agree that young children are able to understand 'simple mental states' such as desires, goals, intentions, 
attention, and perception (for a review see [37]). These skills exhibited by children might not be equivalent to the skills observed in adults. However, Gergely and colleagues (e.g. [18]) suggest that those early skills are crucial for developing the full-fledged mentalistic intentional stance adopted by older children and adults.

\section{Intentional stance and reproduction of cultural norms}

The skills crafted by infants during the first years of life set the foundations of social (and general) cognition. Interacting with others as intentional agents during this critical period helps children determining how others interpret their behavior, how others respond to the behavior and what is expected from them in the future ([5], reviewed by [40]). Furthermore, it enables multiple forms of cultural learning like imitation, instructional learning, use of tools and symbols, and acquisition of language [41]. The development of these cultural tools contribute to the reproduction of the norms that structure human sociality because these are attained through the inter-individual agreement about the statuses of individuals, the entitlements and obligations they entail [42], [43].

The transfer of human cultural tools from generation to generation relies on the intentional stance as the main tool to understand and predict others' behavior. Cognitive development at the early stages of life allows incorporation of societal norms into the children's behavioral repertoire. Using the intentional stance provides the flexibility to interact with others in variable and new social contexts. Importantly, as Michael [41] describes, this process takes place because adults also adopt the intentional stance towards children. For instance, adults set up expectations regarding children's behavior, in acquainting them with culture-specific elements, narratives, practices, social roles, etc. Adults - during the process of enculturalization of children see children as potential rational intentional agents, relying on the intentional stance as the main strategy to predict behavior. In this way, the reference to mental states is consolidated as the crucial foundation and force in social interaction. Additionally, it becomes a mechanism of cultural feedback [41]. Michael also argues that this feedback loop mechanism requires only that young children assume others to have mental states. Once this occurs, a common channel of communication is established between the infants and their caregivers, the channel-mediated through mentalistic vocabulary (thanks to adopting the intentional stance). Furthermore, when infants follow others in adopting the intentional stance, they acquire better interpretational resources, which increases their incorporation into the adult environment, and this, in turn, furthers the process of enculturalization. This is how the intentional stance and cultural learning constitute a feedback loop [41].

Humans are raised immersed in the intentional stance. Extensive training and exposure to the intentional stance starts from very early childhood, and this makes humans experts in this way of explaining and predicting the behaviors of others. Therefore, intentional stance becomes the most available explanatory/ predictive strategy, even when other agents are not necessarily intentional systems. This results in a bias toward viewing agents as having goals, beliefs, and desires and might provide an adaptive heuristic for understanding the world and the agents within. Despite the ease of adopting the intentional stance, humans are still able to identify true intentional systems. People understand that a computer is not tired, that a printer is not reluctant to work or that a volcano is not angry. Rather than assuming that such behavior has been intentionally motivated [42], it is possible that humans interpret behaviors through the filter of "intentional states", as their most familiar, quick and effective way to predict behavior. However, only if this strategy is the most efficient explanatory/predictive strategy, humans continue using it towards a specific agent. If another strategy explains a behavior better, or if they are informed about a better/most efficient strategy, they might change their stance. Intentional stance, however, might be special in that adopting this strategy might trigger a wider set of social behaviors and social attunement, and might create an emotional connection with the observed agents. In summary, we argue that adopting intentional stance is a default, well-trained mechanism, selected by biological and cultural evolution, and a reliable tool in most social interactions with true intentional systems, allowing social attunement.

\section{Empirical evidence for adopting the intentional stance in adults}

Despite the relevance of abilities like mentalizing or attribution of intentionality in daily life, it has proven to be extremely difficult to evaluate them experimentally. It is clear that while people are able to evaluate the intentionality of behavior on demand, they also reason about mental states in a spontaneous manner. Since the early approaches of Heider and Simmel [5], researchers have attempted to understand the factors that trigger mentalizing. Heider \& Simmel [5] presented a series of short animations, each involving two triangles (one large, one small) and a circle, all moving around an empty rectangle. Observers readily attributed 
personality traits to the shapes and described their movements in terms of mental states such as goals and emotions, a finding replicated by subsequent studies (for a review see [43]). The pattern of the movements, rather than properties of the physical appearance of the interacting shapes [43], triggers mentalistic descriptions (i.e. changes in the path, responding to other objects and self-propelled movement). This seminal study showed how readily people adopt the intentional stance even to abstract shapes. However, a question arises regarding the validity of the self-report method used to evaluate the spontaneous adoption of intentional stance. Critics suggest that the design of the experiment and the methods do not allow for ruling out the mediation of other higher-order cognitive mechanisms [43]. Participants' descriptions referring to perceived intentionality might be the result of high order cognitive mechanisms like inference from the questions or the task, rather than the actual observations.

In order to circumvent this caveat, more recently, researchers have chosen tasks with designs that do not require explicit judgement: neuroimaging approaches, complemented with questionnaires and semistructured interviews. A number of neuroimaging studies have explored the neural systems underlying mentalising. This mechanism, closely related to adopting intentional stance and attribution of intentionality, refers to reasoning about others' mental states, in terms of intentions, beliefs and motivations [44]. Numerous tasks have been used to investigate mentalising. Researchers used tasks such as observing and understanding intentions and beliefs of characters in stories ([45-47]) and cartoons ([48], [46], [49]); reporting intentions -or lack thereof- during videos of animated geometrical shapes [10] and predicting competitor's next action in a game [46]. Independently of the task, these studies reported activation in the medial frontal cortex, the superior temporal sulcus (STS) especially around the temporo-parietal junction (TPJ) and the temporal poles adjacent to the amygdala. All of these areas have been identified as part of the mentalizing network. Activity in the STS and TPJ have been also linked to detecting and understanding intended biological motion (e.g. [50], [51], [52]). Moreover, these regions seem to trigger the intentional stance. Some studies revealed activation in these areas related to static images of features that cue intentionality, such as eyes, mouths, hands, and faces (for a review see [50]). Gallagher et al. [53] observed activation in the medial anterior frontal cortex, related to the adoption of the intentional stance. The authors designed an experimental paradigm in which participants played a stone-paper-scissors game in the MRI scanner against agents that were believed to differ in terms of intentional nature (a fellow human, an algorithm using specific rules, or a random number generator). Importantly, participants actually played against pre-programmed sequences in all three conditions. It was participants' beliefs about intentionality that triggered specific activation of the aforementioned brain regions.

In summary, empirical evidence for attribution of intentionality has been a challenge. This is in part due to the difficulty of examining the emergence of attribution of intentionality using self-report measures. New objective methods have shown neural correlates of adoption of the intentional stance. Structures involved in mentalising, such as STS and TPJ, are obviously involved in the general process of adopting the intentional stance.

\section{Intentional stance in social interaction with robots}

In the context of the new societal era, in which robots might soon share our social environments, it is crucial to ask whether humans are likely to adopt the intentional stance towards embodied artificial agents, such as robots. In particular, humanoid robots, as those have some human-like characteristics of appearance. One could speculate that humans would not adopt the intentional stance towards a man-made artefact. In fact, this was confirmed by several findings: a study using a manipulation of the Prisoner's Dilemma [54] showed that areas associated with adopting the intentional stance in the medial prefrontal and left temporoparietal junction were not activated in response to artificial agents, whether or not they were embodied with a human-like appearance. Similarly, Chaminade et al. [55] found that the neural correlates of adopting the intentional stance were not activated in interactions with artificial agents during a relatively simple rockpaper-scissors game. These findings suggest that robots do not naturally induce intentional stance in the human interacting partner.

On the other hand, as discussed earlier, humans are quite eager to attribute mental states and intentions to non-human agents. It is therefore plausible to hypothesize that also in interaction with robots, humans might adopt the intentional stance. In line with this idea, [56] as well as [57] showed that observing goal-directed actions performed by robots, such as grasping a wine glass, evoked similar mirror neuron system activity compared to when observing those actions performed by other humans. This indicates that people interpret 
robots, similarly to other humans, as goal-driven agents. More recently, Thellman et al. [58] presented a series of images and verbal descriptions of different behaviors exhibited either by a person or by a humanoid robot. Participants were asked to rate the intentionality, controllability and desirability of the behaviors, and to judge the plausibility of seven different types of explanations derived from a recently proposed psychological model of lay causal explanation of human behavior. Findings suggest that people adopted the intentional stance toward the robot to a similar degree as in the case of observing other humans.

Very recently, Marchesi et al. (under review) [59] reported that people have a certain tendency to adopt the intentional stance towards humanoid robots, under certain contexts. The authors we developed a questionnaire that explores spontaneous adoption of intentional stance towards a humanoid robot iCub [6o]. This instrument was created in order to evaluate whether people prefer to explain the behavior of iCub using mentalistic or mechanistic terms. The questionnaire consists of 35 fictional scenarios depicting iCub performing different activities. (See Figure 1)

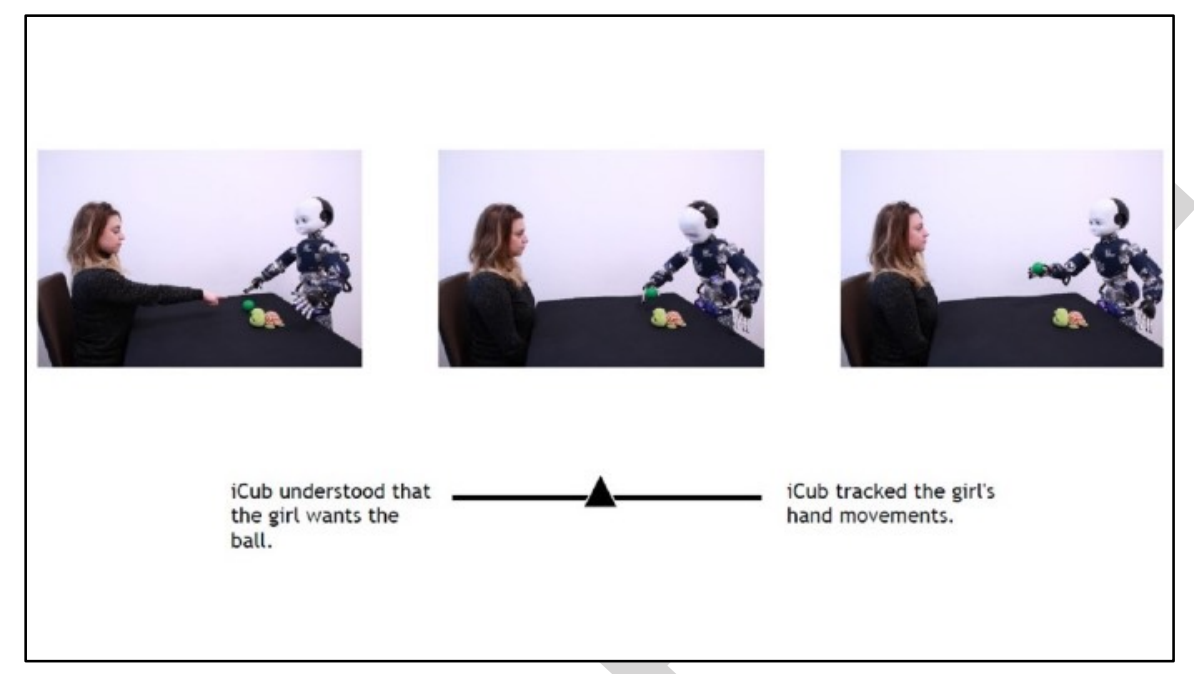

Figure 1. Example of one item of Intentional Stance Questionnaire

.In each scenario, a series of three pictures shows a sequence of events. Participants rate (by moving a slider on a scale) in each scenario if they think iCub's behavior is motivated by a mechanical cause (referring to the design stance, such as malfunctioning, calibration, etc.) or by a mentalistic reason (referring to the intentional stance, such as desire, curiosity, etc.). The slider's scale has a mentalistic description on one extreme, and a mechanistic description on the other. First results with the use of this instrument showed that on average scores had a slight bias toward mechanistic explanations overall, which is not surprising, given that the depicted agent is a robot. This is in line with previous literature that suggests that people attribute a lower degree of intentionality to artificial agents' behavior, compared to other humans ([54], [61], [9]). However, and interestingly, not all the choices in Marchesi et al.'s questionnaire were favouring mechanistic descriptions. Some items of the questionnaire scored predominantly mentalistic descriptions. Furthermore, also individual differences between participants were found, meaning that some participants were more likely to choose mentalistic explanations, while some other participants preferred mechanistic descriptions. Taken together, this suggests that factors such as the human-like appearance of the robot, the context in which the actions unfold, the apparent goal-oriented behavior, as well as individual priors might affect the likelihood of adoption of the intentional stance towards artificial agents.

\section{Factors influencing the likelihood of adopting the intentional stance}

During social interactions, the brain uses highly sensitive mechanisms that are specialized to detect a wide spectrum of signals (i.e. facial expressions, changes in gaze direction). These social signals are in many cases taken as behavioral indicators of mental states. For example, when we see a frown, we infer that it indicates a person's disapproval. Therefore, the presence -or absence- of these indicators might be a crucial factor in the likelihood of adopting the intentional stance. Hence, determining the specific parameters of those 
indicators that facilitate the adoption of the intentional stance is important and informative with respect to design and implementation of behavior in artificial agents.

Research in HRI shows that implementing human-like characteristics in artificial agents facilitate social interaction (see [62] and [63] for review). Robots that show human-like design and behavior are more accepted ([64-66]), more pleasant [67], are perceived as more usable [68], are easier to get acquainted with [69], and are more engaging [70], relative to purely functional designs. Furthermore, robots that exhibit social signals like facial expressions [71], emotions [72] or respect turn taking in a conversation [73] were more likable and produced more emotional responses in participants. More recently, Willemse at al. [74] showed, in a gaze-leading paradigm, that participants anthropomorphized and liked more those robots which followed the participants' gaze. In one condition of the experiment, a robot avatar looked $80 \%$ of the time at the same object that participants chose, whereas in another condition the robot was more likely $(80 \%$ of time) to look at a different object. Participants reported preferring the robot that followed their object preference and rated it as more human-like and as more likeable, relative to the one that did not follow the participant's gaze. Kompatsiari et al. [75] showed that mutual gaze established by a humanoid robot affected participants' judgments of human-likeness of the robot. Wykowska et al. [76] showed that variability in temporal characteristics of gaze behavior was perceived as human-controlled, despite that the behavior was executed by a robotic agent (the iCub [6o]).

In summary, it seems that people might use various behavioral signals of an observed agent as hints that encourage adopting the intentional stance or ascribing humanness towards the agent.

\section{Consequences of adopting the intentional stance}

Understanding the conditions under which humans adopt the intentional stance towards artificial agents is not only of theoretical significance but might also have implications in terms of social interaction. Adopting the intentional stance towards an artificial agent might have multi-faceted consequences with respect to social attunement with the agent. We propose the term "social attunement" as an umbrella concept which encompasses all mechanisms of social cognition (e.g., mutual gaze, joint attention, or spatial perspective taking) activated during social interactions.

In line with the idea that adopting intentional stance might impact mechanisms of social cognition, Wiese et al. [77] showed that people were more inclined to engage in joint attention with a robot when they believed its behavior represented an intentional system. The authors used a gaze-cueing paradigm with robot and humans faces. In three experiments, they instructed participants differently regarding the agency of the observed behavior. In the first experiment, without belief manipulation, human faces evoked larger gaze cueing effect compared to the robot faces. In the second and third experiment, participants were told that the observed gaze behavior was the result of either mental operations (gaze behavior controlled by a human) or was produced by an algorithm. Results showed larger gaze cueing effects when participants believed that they were observing human-controlled behavior (independent of whether they observed a human or a robot face), relative to the algorithm-generated behavior. Furthermore, Wykowska et al. [78] examined the electrophysiological correlates of this modulation of the gaze-cueing effect. Findings revealed larger amplitude of the P1 component of the EEG signal (time window between 100-140 ms, locked to target presentation) for validly cued, versus invalidly cued targets when the behavior was believed to be controlled by a human. This difference in amplitude was absent when participants believed the behavior of the observed face was generated by an algorithm. Therefore, the P1 effect mirrored behavioral findings observed in Wiese et al. [77] and showed that the behavioral effects are due to early attention mechanisms. In sum, already early attention mechanisms are modulated by whether participants adopt an intentional or a design stance towards an agent.

Collectively, these findings provide support to the notion that adopting intentional stance might influence the interpretation of basic social signals, and as a consequence, activation of fundamental mechanisms of social cognition.

\section{Ethical considerations}

Social attunement is a crucial factor in social interactions, and therefore it seems to also be indispensable for smooth and natural HRI. If adopting the intentional stance indeed facilitates social attunement, then it would be beneficial to design robots and robot behaviors that evoke adoption of intentional stance. However, this might have some potentially controversial implications. Humans are already attached to a number of 
artefacts. Technology has made mobile phones, computers and video games extremely engaging, taking advantage of human cognitive resources. Attention-capturing colorful blinking apps, ringing tones, frequent presentation of publicity banners on a webpage and the reward-oriented notifications from social media, have all been designed to hijack hard-wired psychological mechanisms. This facilitates incorporation of new technologies in people's lives, but with a drawback of making those services and products indispensable and highly addictive. In this context, it is crucial to discuss the societal implications of robots that are highly attuned to the human cognitive mechanisms. Robots that are engaging, that learn and understand human behavior and that attune socially with humans could be used in extremely beneficial manner as assistants and supporters in daily activities. It is very likely that such social robots could generate emotional attachment or other types of bonds that might not have been yet studied. It is unclear at this point whether this type of attachment has negative or positive consequences for humans. Therefore, it is crucial to discuss and define what type of social bonding with a robot is desired and perhaps use the very same technology to avoid any negative consequences. For example, robots could be designed to detect when patterns of addictive behavior arise and mitigate its possible escalation.

In general, the societal implications of introducing social robots that are very well attuned to humans is a very delicate topic which requires being addressed with a serious and rigorous approach from philosophy, psychology, anthropology and engineering. Unfortunately, like in other occasions where humans have to face a significant societal change, many fears arise. In a viral fashion, these fears are broadcasted often without a critical, rational approach. The topics of artificial intelligence and robotics of the future are all over the news and media. Often these reports bring about unrealistic science-fiction-like scenarios for a technology that is still far from being as advanced as depicted. In this context, it is the task of researchers to critically evaluate the potential implications of the introduction of robots into human daily environments, and take adequate measures to prevent negative consequences, if any.

Overall, it is important to focus research, and public outreach of research, on the potential advantages that the new robotic technologies might bring to society. Robots equipped with well-designed social mechanisms would be able to adapt to a wide variety of contexts. One of the areas that robots are already shown to be very useful is the robot-assisted training of social skills for children diagnosed with autism spectrum disorder (ASD). According to several studies (for a review see [79]), individuals with ASD open up to interaction with robots, with which they can train their social behaviors and skills, such as emotion recognition or joint attention. Most importantly, those skills seem to generalize from interaction with robots to interaction with other humans ([80-82]). Apart from designing robots designated to an entire type of population, one could think about robots that adapt socially to individual needs, providing a custom-made level of social engagement, without compromising functionality. For example, if a person does not seem interested in establishing a human-like interaction with the robot, the robot would recognize this attitude and adapt its communicative style (e.g., a minimal degree of eye contact, limited use of language and less spontaneous behavior). On the other hand, if a robot would detect signals from the human that invite more social contact, the robot could increase its level of socially engaging behaviors. Robots with such skills could take care of the elderly, be placed at information desks, or assist with certain tasks at home.

In summary, we propose that developing socially capable robots could have an extremely positive impact on society. However, implementation of social skills in this type of artificial agents should take into consideration possible emotional and psychological consequences. Therefore, an extensive and in-depth interdisciplinary debate with rational and critical evaluation of the state-of-the-art technology and its implications is indispensable. This debate should also involve the public and should be made open and very transparent.

\section{Future directions}

In our approach (Kompatsiari et al. [83]; Schellen et al. [84]; Wiese, Metta \& Wykowska [9], see also: www.instanceproject.eu), we stress that in examining social attunement between humans and robots one needs to use the methods of experimental psychology and cognitive neuroscience in natural (but experimentally controlled) human-robot interaction protocols. This allows for targeting and isolating specific cognitive mechanisms of the human brain and designing robots that evoked those specific mechanisms. Proper experimental control allows for better reproducibility and generalizability of results. One of the challenges is to translate well-established paradigms of experimental psychology into more naturalistic interaction scenarios [83]. This is because classical experimental protocols often present to participants 
reduced (in naturalness) stimuli, such as schematic drawings of faces. This is done in order to maintain experimental control. However, having already established robust replicable effects with the use of such stimuli, we can now move towards more ecologically valid protocols while still trying to maintain experimental control. This is precisely our approach. Once we succeed in transferring the standard protocols of experimental psychology into human-robot interaction studies, we will be able to understand whether, and under what conditions, the well-studied mechanisms of human social cognition are evoked in human-robot interaction. By the same token, it is crucial to investigate objective (and perhaps implicit) measures of when people adopt the intentional stance towards robots. So far, we have developed a more explicit measure, based on self-report, the Intentional Stance questionnaire [59], but future studies will be dedicated to discovering neural (and behavioral) correlates of subjective reports which indicate mentalistic or mechanistic mode of explaining the observed behavior. Once such markers are identified, we will be able to determine -with objective implicit measures- whether, during an interaction with a robot, a participant is in the intentional or design stance mode. It might be beneficial for the interaction to online adjust behavior of the robot in order to elicit one or the other stance, dependent on the current needs (if a robot needs to be received as a social entity, it might modify behavior to evoke adoption of intentional stance, or, if it needs to be perceived instrumentally as a tool for a given task, it might behave in a manner that evokes the adoption of the design stance). Such online adaptation of behavior is of course still in the realm of fantasy, but the goal of identifying neural or behavioral correlates of adopting the intentional stance is within the research agenda for the near future.

\section{Conclusions}

Artificial agents, and humanoid robots specifically, are likely to be increasingly present in the human social environment. It is plausible that designing the robots in a way that evokes adoption of intentional stance towards them might facilitate social attunement and their integration into society. We postulate that in order to make this happen in a proper way, we need to use the methods of experimental psychology and cognitive neuroscience in natural, but experimentally controlled, human-robot interaction protocols. In the process of developing robots with social capabilities that attune well with humans and potentially evoke adoption of intentional stance, we should actively discuss societal impact of such technology, and address potential issues with reason and appropriate scientific methods.

\section{Acknowledgements}

Work on this chapter, and research agenda described in "Future directions" section of the chapter have been supported by the European Research Council under the European Union's Horizon 2020 research and innovation program (grant awarded to Agnieszka Wykowska, titled "InStance: Intentional Stance for Social Attunement”. ERC starting grant, grant agreement No.: 715058)

\section{References}

1. Dennett, D. C. (1987) The Intentional Stance. MIT Press.

2. Dennett, D. C. (1971) 'Intentional systems', Journal of Philosophy. Bradford Books, 68(February).

3. Dennett, D. (2009) 'Intentional Systems Theory', in The Oxford Handbook of Philosophy of Mind. doi: 10.1093/oxfordhb/ 9780199262618.003.0020.

4. Gray, H. M., Gray, K. and Wegner, D. M. (2007) ‘Dimensions of Mind Perception', Science, 315(5812), p. 619 LP-619.

5. Heider, F., \& Simmel, M. (1944). An experimental study of apparent behaviour. American Journal of Psychology, 57, 243-259.

6. Epley, N., Waytz, A. and Cacioppo, J. T. (2007) 'On Seeing Human: A Three-Factor Theory of Anthropomorphism', Psychological Review, 114(4), pp. 864-886. doi: 10.1037/0033-295X.114.4.864.

7. Mitchell, R.W., Thompson, N. S., Miles, H. L. (1997) 'Anthropomorphism, Anecdotes, and Animals', Current Anthropology.

8. Waytz, A., Epley, N. and Cacioppo, J. T. (2010) 'Social cognition unbound: Insights into anthropomorphism and dehumanization', Current Directions in Psychological Science. Doi: 10.1177/0963721409359302.

9. Wiese, E., Metta, G. and Wykowska, A. (2017) 'Robots as intentional agents: Using neuroscientific methods to make robots appear more social', Frontiers in Psychology. doi: 10.3389/fpsyg.2017.01663.

10. Castelli, F., Happé, F., Frith, U., and Frith, C. 2000. Movement and mind: A functional imaging study of perception and interpretation of complex intentional movement patterns. NeuroImage 12: 314-325. 
11. Iacoboni, M. et al. (2004) 'Watching social interactions produces dorsomedial prefrontal and medial parietal BOLD fMRI signal increases compared to a resting baseline', NeuroImage. doi: 10.1016/j.neuroimage.2003.11.013.

12. Wimmer, H. and Perner, J. (1983) 'Beliefs about beliefs: Representation and constraining function of wrong beliefs in young children's understanding of deception', Cognition. Doi: 10.1016/0010-0277(83)90004-5.

13. Griffin, R. and Baron-Cohen, S. (2002) 'The intentional stance: developmental and neurocognitive perspectives', Daniel Dennett.

14. Apperly, I. (2011) Mindreaders: The cognitive basis of 'theory of mind', Mindreaders: The cognitive basis of 'theory of mind'. New York, NY, US: Psychology Press.

15. Woodward, A. L. (1998) 'Infants selectively encode the goal object of an actor's reach', Cognition. doi: 10.1016/ Soo10-0277(98)00058-4.

16. Senju, A., Csibra, G. and Johnson, M. H. (2008) 'Understanding the referential nature of looking: infants' preference for object-directed gaze.', Cognition, 108(2), pp. 303-19. doi: 10.1016/j.cognition.2008.02.009.

17. Stern, D. N. (1998) 'The Interpersonal World of the Infant: A View from Psychoanalysis and Developmental Psychology', New York Basic. doi: 10.1017/CBO9781107415324.004.

18. Gergely, G. and Csibra, G. (2003) 'Teleological reasoning in infancy: The naïve theory of rational action', Trends in Cognitive Sciences. doi: 10.1016/S1364-6613(03)00128-1.

19. Ma, L. and Lillard, A. S. (2006) 'Where is the real cheese? Young children's ability to discriminate between real and pretend acts', Child Development. doi: 10.1111/j.1467-8624.2006.00972.x.

20. Behne, T. et al. (2005) 'Unwilling versus unable: Infants' understanding of intentional action', Developmental Psychology. Doi: 10.1037/0012-1649.41.2.328.

21. Repacholi, B. M. and Gopnik, A. (1997) 'Early reasoning about desires: evidence from 14- and 18-month-olds.', Developmental psychology. Doi: 10.1037/0012-1649.33.1.12.

22. Tomasello, M. et al. (2005) 'Understanding and sharing intentions: The origins of cultural cognition', Behavioral and Brain Sciences. doi: 10.1017/S0140525X05000129.

23. Baldwin, D. A. et al. (2001) 'Infants parse dynamic action', Child Development. Doi: 10.1111/1467-8624.00310.

24. Sorce, J. F. et al. (1985) 'Maternal Emotional Signaling. Its Effect on the Visual Cliff Behavior of 1-Year-Olds', Developmental Psychology. Doi: 10.1037/0012-1649.21.1.195.

25. Feinman, S. and Lewis, M. (1983) 'Social referencing at ten months: a second-order effect on infants' responses to strangers.', Child development. doi: 10.1111/j.1467-8624.1983.tbo0509.x.

26. Johnson, S., Slaughter, V. and Carey, S. (1998) 'Whose gaze will infants follow? The elicitation of gaze following in 12-montholds', Developmental Science. Doi: 10.1111/1467-7687.00036.

27. Carpenter, M., Akhtar, N. and Tomasello, M. (1998) 'Fourteen- through 18-month-old infants differentially imitate intentional and accidental actions', Infant Behavior and Development. doi: 10.1016/So163-6383(98)900o9-1.

28. Meltzoff, A. N. (1995) 'Understanding the Intentions of Others: Re-Enactment of Intended Acts by 18-Month-Old Children', Developmental Psychology. Doi: 10.1037/0012-1649.31.5.838.

29. Tomasello, M., Kruger, A. C. and Ratner, H. H. (1993) 'Cultural learning', Behavioral and Brain Sciences. doi: 10.1017/ S0140525X0003123X.

30. Harris, P. (1993). Pretending and planning. In S. Baron-Cohen, H. Tager- Flusberg, \& D. Cohen (Eds.), Understanding other minds: perspectives from autism. Oxford: Oxford University Press.

31. Wellman, H. M., Cross, D. and Watson, J. (2001) 'Meta-analysis of theory-of-mind development: The truth about false belief', Child Development. Doi: 10.1111/1467-8624.00304.

32. Bartsch, K. and Wellman, H. M. (1995) Children talk about the mind. New York, NY, US: Oxford University Press.

33. Lillard, A. S. (1998). Wanting to be it: Children's understanding of intentions underlying pretense. Child Development, 69 , 981-993.

34. Schult, C. A. (2002) Children's understanding of the distinction between intentions and desires. Child Development 73:1727-47.

35. Perner, J. (1991) Understanding the Representational Mind. MIT Press.

36. Baird, J. A. and Moses, L. J. (2001) 'Do Preschoolers Appreciate That Identical Actions May Be Motivated by Different Intentions?', Journal of Cognition and Development. doi: 10.1207/S15327647JCD0204_4.

37. Johnson, S. C. (2003) 'Detecting agents', Philosophical Transactions of the Royal Society B: Biological Sciences. doi: 10.1098/rstb.2002.1237. 
38. Mar, R. A. and Neil Macrae, C. (2008) 'Triggering the Intentional Stance', Empathy and Fairness, pp. 111-120. doi: 10.1002/9780470030585.ch9.

39. Dennett, D. C. (1997) 'True Believers: The Intentional Strategy and Why It Works', Mind Design. doi: 10.1007/ s13398-014-0173-7.2.

40. Malle, B. F. (2011) 'Attribution Theories: How People Make Sense of Behavior', Theories in Social Psychology., pp. 72-95.

41. Michael, J. (2015) 'The intentional stance and cultural learning: A developmental feedback loop', in Content and Consciousness Revisited. Doi: 10.1007/978-3-319-17374-0_9.

42. Searle, J. R. (1995) Construction of Social Reality, The Free Press.

43. Scholl, B. J. and Tremoulet, P. D. (2000) 'Perceptual causality and animacy', Trends in Cognitive Sciences, 4(8), pp. 299309. doi: 10.1016/S1364-6613(00)01506-0.

43. Gilbert, M. (1990) 'Walking Together: A Paradigmatic Social Phenomenon', Midwest Studies In Philosophy. doi: 10.1111/ j.1475-4975.1990.tboo202.x.

44. Frith, C. and Frith, U. (2000) How we predict what other people are going to do. Brain Res. 1079(1):36-46

45. Fletcher, P. C. et al. (1995) 'Other minds in the brain: A functional imaging study of "theory of mind" in story comprehension', Cognition. doi: 10.1016/o010-0277(95)oo692-R.

46. Gallagher, H. L., Happe, F., Brunswick, N., Fletcher, P. C., Frith, U., and Frith, C. D. 200o. Reading the mind in cartoons and stories: An fMRI study of "theory of mind" in verbal and nonverbal tasks. Neuropsychologia 38: 11-21.

47. Saxe, R. and Kanwisher, N. (2013) 'People thinking about thinking people: The role of the temporo-parietal junction in "theory of mind”, in Social Neuroscience: Key Readings. Doi: 10.4324/9780203496190.

48. Brunet, E. et al. (2000) 'A PET investigation of the attribution of intentions with a nonverbal task', NeuroImage. doi: 10.1006/nimg.1999.0525.

49. Vogeley, K. et al. (2001) 'Mind reading: Neural mechanisms of theory of mind and self-perspective', NeuroImage. doi: 10.1006/nimg.2001.0789.

50. Allison, T., Puce, A. and McCarthy, G. (2000) 'Social perception from visual cues: Role of the STS region', Trends in Cognitive Sciences. doi: 10.1016/S1364-6613(00)01501-1.

51. Pelphrey, K. A., Morris, J. P. and McCarthy, G. (2004) Grasping the intentions of others: the perceived intentionality of an action influences activity in the superior temporal sulcus during social perception, Journal of cognitive neuroscience. Doi: 10.1162/0898929042947900.

52. Saxe, R. et al. (2004) 'A region of right posterior superior temporal sulcus responds to observed intentional actions', Neuropsychologia, 42(11), pp. 1435-1446. doi: 10.1016/j.neuropsychologia.2004.04.015.

53. Gallagher, H., Jack, A., Roepstorff, A., and Frith, C. (2002). Imaging the intentional stance in a competitive game. Neuroimage 16, 814 .

54. Krach, S. et al. (2008) 'Can machines think? Interaction and perspective taking with robots investigated via fMRI', PLoS ONE. doi: 10.1371/journal.pone.0002597.

55. Chaminade, T., Rosset, D., Da Fonseca, D., Nazarian, B., Lutcher, E., Cheng, G., \& Deruelle, C. (2012). How do we think machines think? An fMRI study of alleged competition with an artificial intelligence. Frontiers in Human Neuroscience, 6 , 103. http://doi.org/10.3389/fnhum.2012.00103

56. Gazzola, V. et al. (2007) 'The anthropomorphic brain: The mirror neuron system responds to human and robotic actions', NeuroImage. doi: 10.1016/j.neuroimage.2007.02.003.

57. Oberman, L. M. et al. (2007) 'EEG evidence for mirror neuron activity during the observation of human and robot actions: Toward an analysis of the human qualities of interactive robots', Neurocomputing. doi: 10.1016/j.neucom.2006.02.024.

58. Thellman, S., Silvervarg, A. and Ziemke, T. (2017) 'Folk-psychological interpretation of human vs. humanoid robot behavior: Exploring the intentional stance toward robots', Frontiers in Psychology, 8(NOV), pp. 1-14. doi: 10.3389/fpsyg.2017.01962.

59. Marchesi et al. (under review) Do we adopt the intentional stance towards humanoid robots?

6o. Metta, G. et al. (2008) 'The iCub humanoid robot: an open platform for research in embodied cognition', in Performance Metrics for Intelligent Systems Workshop (PerMIS 2008).

61. Chaminade, T. et al. (2010) 'Brain Response to a Humanoid Robot in Areas Implicated in the Perception of Human Emotional Gestures', PLOS ONE. Public Library of Science, 5(7), p. e11577. doi.org/10.1371/journal.pone.o011577.

62. Fink, J. (2012) 'Anthropomorphism and human likeness in the design of robots and human-robot interaction', Lecture Notes in Computer Science (including subseries Lecture Notes in Artificial Intelligence and Lecture Notes in Bioinformatics), 7621 LNAI, pp. 199-208. doi: 10.1007/978-3-642-34103-8_20. 
63. Fong, T., Nourbakhsh, I., \& Dautenhahn, K. (2003) 'A Survey of Socially Interactive Robots: Concepts, Design, and Applications. Robotics and autonomous systems. doi: 10.1016/So921-8890(02)00372-X.

64. Venkatesh, V. and Davis, F. D. (2000) 'A Theoretical Extension of the Technology Acceptance Model: Four Longitudinal Field Studies', Management Science. doi: 10.1287/mnsc.46.2.186.11926.

65. Duffy, B.R.: Anthropomorphism and the social robot. Robotics and Autonomous Sys- tems 42, 177-190 (2003)

66. Goetz, J. and Kiesler, S. (2002) 'Cooperation with a robotic assistant', in CHI 'o2 extended abstracts on Human factors in computing systems - CHI'O2. doi: 10.1145/506443.506492.

67. Axelrod, L. and Hone, K. (2005) 'E-motional advantage: performance and satisfaction gains with affective computing', Proceedings of ACM CHI 2005 Conference on Human Factors in Computing Systems. Doi: 10.1145/1056808.1056874.

68.Riek,L.D.,Rabinowitch,T.-C.,Chakrabarti,B.,Robinson,P.:How anthropomorphism affects empathy toward robots. In: Proceedings of the 4th ACM/IEEE International Conference on Human Robot Interaction, pp. 245-246. ACM, New York (2009)

69. Hegel, F. et al. (2008) 'Understanding Social Robots: A User Study on Anthropomorphism', in Robot and Human Interactive Communication, 2008. RO-MAN 2008. The 17th IEEE International Symposium on. doi: 10.1109/ ROMAN.2008.4600728.

70. Bartneck, C., Forlizzi, J.: Shaping human-robot interaction: understanding the social as- pects of intelligent robotic products. In: CHI 2004 Extended Abstracts on Human Factors in Computing Systems, pp. 1731-1732. ACM, New York (2004)

71. Eyssel, F., Hegel, F., Horstmann, G., Wagner, C.: Anthropomorphic inferences from emotional nonverbal cues: A case study. In: 2010 IEEE RO-MAN, pp. 646-651. IEEE (2010)

72. Gonsior, B. et al. (2011) 'Improving aspects of empathy and subjective performance for HRI through mirroring facial expressions', in Proceedings - IEEE International Workshop on Robot and Human Interactive Communication. doi: 10.1109/ROMAN.2011.6005294.

73. Fussell, S.R., Kiesler, S., Setlock, L.D., Yew, V.: How people anthropomorphize robots. In: Proceedings of the 3rd ACM/IEEE International Conference on Human Robot Interac- tion, pp. 145-152. ACM, New York (2008)

74. Willemse, C., Marchesi, S. and Wykowska, A. (2018) 'Robot faces that follow gaze facilitate attentional engagement and increase their likeability', Frontiers in Psychology. doi: 10.3389/fpsyg.2018.00070.

75. Kompatsiari, K. et al. (2017) 'The Importance of Mutual Gaze in Human-Robot Interaction BT - Social Robotics', in Kheddar, A. et al. (eds). Cham: Springer International Publishing, pp. 443-452.

76. Wykowska, A. et al. (2015) 'Humans are Well Tuned to Detecting Agents Among Non-agents: Examining the Sensitivity of Human Perception to Behavioral Characteristics of Intentional Systems', International Journal of Social Robotics. doi: 10.1007/s12369-015-0299-6.

77. Wiese, E. et al. (2012) 'I See What You Mean: How Attentional Selection Is Shaped by Ascribing Intentions to Others', PLoS ONE, 7(9). doi: 10.1371/journal.pone.0045391.

78. Wykowska, A., Wiese, E., Prosser, A., Müller, H.J. (2014). Beliefs about the minds of others influence how we process sensory information. PLOS ONE, 9 (4), e94339

79. Cabibihan, J.J., Javed, H., Ang, M. et al. (2013) Why Robots? A Survey on the Roles and Benefits of Social Robots in the Therapy of Children with Autism. International Journal of Social Robotics, 5: 593. https://doi.org/10.1007/ s12369-013-0202-2

80. Wykowska, A. et al. (2015) 'Autistic traits and sensitivity to human-like features of robot behavior', Interaction Studies. doi: 10.1075/is.16.2.09wyk.

81. Kajopoulos, J. et al. (2015) 'Robot-Assisted Training of Joint Attention Skills in Children Diagnosed with Autism', in Social Robotics: Proceedings of the 7th International Conference on Social Robotics, ICSR 2015, Paris, France. Doi: 10.1007/978-3-319-25554-5_30.

82. Dautenhahn, K. (2007). Socially intelligent robots: dimensions of human-robot interaction. Philos. Trans. R. Soc. Lond. B Biol. Sci. 362, 679-704.

83. Kompatsiari, K., Pérez-Osorio, J., Metta, G., Wykowska, A. (in press) Neuroscientifically-grounded research for improved human-robot interaction.

84. Schellen, E., Pérez-Osorio, J., Wykowska, A. (in press) Social Cognition in Human-Robot Interaction: Putting the 'H' back in 'HRI' 